\title{
Staphylococcus aureus Masking Tuberculous Joint Infection
}

\author{
Mohammed A. Shaikhomer, MD, MPH \\ 'Department of Internal Medicine, King Abdulaziz University, Jeddah, Saudi Arabia \\ ${ }^{2}$ Department of Infectious Disease, University of Miami-Miller School of Medicine, \\ Jackson Memorial Hospital, Miami, Florida, USA
}

\section{Correspondence \\ Dr. Mohammed A. Shaikhomer \\ 300 S. Biscayne Blvd. Apt 1414 \\ Miami, FL 33131 U.S.A. \\ e.M: oshaikhomer@kau.edu.sa \\ Submission: 10 September 2017 \\ Accepted: 19 September 2017}

\section{Citation}

Shaikhomer M. Staphylococcus aureus masking tuberculous joint infection. JKAU Med Sci 2017; 24 (4): 41-45. DOI: 10.4197/Med. 24.4.6

\begin{abstract}
Skeletal tuberculosis accounts for around 10 percent of cases of extrapulmonary tuberculosis. Hip and knee involvement are the most commonly affected sites after spine. Clinical manifestations include swelling, pain, and loss of joint function that progress over weeks to months. In the setting of tuberculous arthritis, local soft tissue swelling, osteopenia, and bone destruction (with relative preservation of cartilage space) occur early in the disease. Subsequent findings include structural collapse, sclerotic changes, and soft tissue calcification. Delay in diagnosing tuberculous arthritis can lead to functional disability and poor outcome. Superimposed infection with another organism occurs very rarely and can lead to delay in diagnosing the condition. We present a case of tuberculous knee arthritis that was superimposed with Staphylococcus aureus infection. The low index of suspicion at the initial presentation together with the superimposed infection led to a delayed diagnosis. Our patient's risk factor was immigration from a country endemic with tuberculosis. The case was diagnosed 32 months from initial presentation. In the presence of clinical and epidemiological risk for tuberculous arthritis, requesting the appropriate tests is crucial to avoid any diagnostic delay.

Keywords

Mycobacterium tuberculosis; Tuberculous arthritis; Knee joint infection; Methicillin-resistant Staphylococcus aureus
\end{abstract}

\section{Introduction}

$T$ he development of the various advanced tests to diagnose tuberculosis (TB) has led to early diagnosis of the disease. These tests include: the use of special stain; culture; polymerase chain reaction (PCR); tissue biopsy; radiological finding; QuantiFERON test; and purified protein derivative (PPD) testing. Skeletal TB represents around 10\% of extra-pulmonary TB cases $^{[1,2]}$. Knee involvement represents the third most common skeletal site after spine and hip joints ${ }^{[2,3]}$. Superimposed infection with another bacterial pathogen is rare and may lead to delays in diagnosing the condition ${ }^{[3-5]}$. The pathophysiology of skeletal TB is usually due to reactivation of an old TB infection in a primary site, which then seeds the bone or joint via the bloodstream. The risk of TB reactivation in immigrants from an endemic country persists for many years ${ }^{[6]}$. In the appropriate clinical and epidemiological setting for skeletal TB, requesting stain, $P C R$, and culture for acid-fast bacilli is crucial to avoid diagnostic delays. Synovial biopsy can help in case of negative stain, PCR and culture results. QuantiFERON and PPD tests are used to diagnose latent TB and sometimes can help in diagnosing active disease. 
We report a case of tuberculous arthritis of the knee superimposed with Staphylococcus aureus (S. aureus) infection. The growth of S. aureus, together with the low index of suspicion at the initial presentation, has led to delay in diagnosing TB.

\section{Case Report}

A 67-year old female presented to the orthopedic clinic at (Jackson Memorial Hospital, Miami, FL USA) with a 2-year history of chronic left knee pain. She was diagnosed initially with osteoarthritis. The patient denied any fever, weight loss, or night sweats. Past medical history includes hypertension managed with an anti-hypertensive agent. She has no prior history of surgery or significant trauma to the knees. The patient is originally from Vietnam and immigrated to the United States ten years ago. Since then, she has not traveled outside of Florida. Her daughter reports that the patient had a negative purified protein derivative (PPD) test when they initially moved to the United States. The patient reports no contact with a patient who has TB in the past. Initial evaluation with $\mathrm{X}$-ray revealed severe tricompartmental degenerative changes of the left knee with small suprapatellar effusion and no fracture. The patient was lost in follow up and presented again after one year with an increase in pain intensity in the last three months which resulted in limping and difficulty walking. Her pain was accompanied by swelling. A repeated X-ray showed interval progression of the tricompartmental osteoarthritis with moderate to large left suprapatellar effusion. Further workup with magnetic resonance imaging (MRI) showed destructive arthritic changes with fragmentation and collapse of the subchondral surfaces with multifocal periarticular erosions, effusion, and diffuse synovitis. At that time, the differential diagnosis was inflammatory arthropathy versus chronic septic arthritis. A knee arthrocentesis was performed and sent for analysis. It revealed bloody fluid with a total cell count of 2222 , neutrophil $25 \%$, lymphocyte $75 \%$, and red blood cells of 155556 . Fungal and bacterial cultures were negative. Acid-fast bacilli (AFB) stain was negative, however AFB culture was not sent. There were no fluid crystals. Complete blood count showed white blood cell count of 7 with normal differential and Hemoglobin of 11.2. Otherwise, all basic blood tests were within normal limits. The patient was referred to the rheumatology clinic. Further blood workup revealed negative antinuclear antibody (ANA) and anti-cyclic citrullinated peptide (CCP). Her uric acid was within the normal limit. C-reactive protein (CRP) is $1.7 \mathrm{mg} / \mathrm{dl}$ and erythrocyte sedimentation rate (ESR) is $50 \mathrm{~mm} / \mathrm{hr}$. She was treated with a nonsteroidal antiinflammatory agent and had a local steroid injection for pain relief. After four weeks, follow-up in the clinic showed persistent pain and difficulty in walking. At that time, a decision was made to proceed with surgery for a total knee arthroplasty. However, on the day of surgery a purulent drainage ulcer was noticed over the posterior medial aspect of the left calf. The procedure was canceled; the patient was sent home on Bactrim orally for two weeks with a follow up appointment in the clinic. At that time, fluid from the draining wound was not sent for cultures. Two months later, follow up MRI showed severe fibro-inflammatory process throughout the knee joint with severe erosive changes at the distal femur, proximal tibia and articular surface of the patella (Fig. 1). The patient subsequently underwent incision and drainage with total knee arthroplasty and antibiotic spacer placement. Intraoperative fluids were sent for bacterial, fungal and AFB cultures. The intraoperative

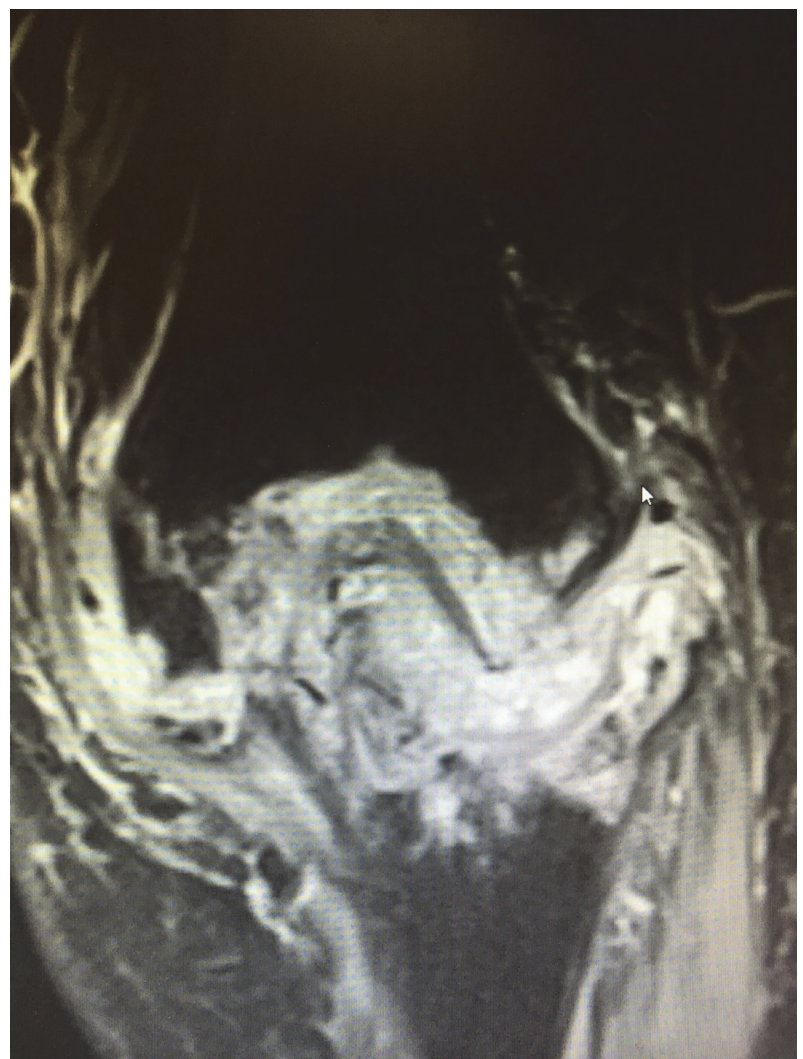

Figure 1. Magnetic resonance imaging of the patient's left knee joint showing severe fibro-inflammatory process throughout the knee joint with severe erosion of the distal femur and proximal tibia as well as the articular surface of the patella. There is edema throughout the adjacent soft tissues. There is severe derangement and destruction of all the intra-articular structures. 
bacterial cultures grew methicillin-resistant S. aureus, Infectious disease consultation was obtained, and the patient was treated with intravenous vancomycin for a total of 6 weeks. At the end of the antibiotic therapy, she had some improvement regarding pain but still had significant knee swelling. Finally, AFB cultures grew Mycobacterium TB which was drug susceptible. The patient was started on anti-TB therapy and had significant improvement of the pain and swelling later. Her functional status was improving with TB medications and physical therapy. She now can walk with a cane and requires intermittent pain medications after a total of 12 months of anti-TB therapy. The duration of TB medications was prolonged due to the extensive bone and joint damage. She continues to have persistent stiffness of the joint with an inability to fully flex the knee. She did not have any further surgical intervention and has now been working with physical therapist for three months since finishing the TB therapy.

\section{Discussion}

Tuberculosis arthritis represents a challenging diagnosis most of the time. In the right clinical and epidemiological context, physician should request AFB stain and cultures. Sometimes synovial biopsies are needed to reach the diagnosis. The difficulty and delay in diagnosing skeletal TB are well established. The average delay in diagnosing the disease is between 16 and 19 months ${ }^{[7,8]}$. In our case, the delay in diagnosis was due to multiple factors. First, the index of suspicion at the initial presentation was low. Second, the first fluid aspirate was sent only for AFB stain and not AFB culture. Lastly, the growth of S. aureus in the intraoperative fluid aspirate acted like the culprit, but in fact, was likely a superimposed infection after the steroid injection, given there was no bacterial growth in the initial fluid aspirate. Steroid injection can have adverse outcomes including infection. Local steroid injection should be used with caution especially in cases with ambiguous diagnosis. The diagnosis of our case was established after 32 months from the initial presentation.

The high index of suspicion at the initial presentation is an important factor to avoid diagnostic delay. In the presence of high clinical suspicion, synovial fluid should be sent for AFB stain, PCR and culture. Synovial biopsy should be considered if AFB studies are negative. In our case, PCR, synovial biopsy, and QuantiFERON tests were not used to diagnose the condition. Those tests can help in diagnosing the condition earlier instead of awaiting culture results. In the presence of high clinical suspicion, anti-TB medications can be started early while awaiting final cultures and pathology results.

There are many risk factors which can lead to the development of TB. These factors include history of previous TB infection, recent TB contact, immigration from a TB endemic area, low socioeconomic status, homelessness, steroid therapy, and immunodeficiency ${ }^{[9]}$. Our patient was an immigrant from Vietnam, which is considered one of the 30 high burden countries for $\mathrm{TB}^{[10]}$. Per her daughter, she had a negative PPD test upon entry to the United States. Purified protein derivative can sometimes be falsely negative ${ }^{[11]}$. Tuberculosis can potentially reactivate anytime in life after a period of latency ${ }^{[6,11]}$. Finally, imaging studies can help in diagnosing TB by raising the clinical suspicion. At the initial stages, TB causes synovitis with or without bony erosions. At a later stage, narrowing of the joint space with progressive destruction to the bone and cartilage is observed ${ }^{[12]}$. Cold abscess and draining sinuses often develop in chronic cases. For cases diagnosed at early stages, TB therapy alone results in a complete resolution. Surgery is necessary only when severe joint instability requires fusion, and if therapy with TB medications has failed. Our patient had progressive destruction of the cartilage and bone on images but an overall improvement after TB therapy, total knee arthroplasty, and surgical drainage. Her TB therapy was prolonged to a total of 12 months due to the extensive bony and tissue destruction. She required no further surgical intervention after the treatment. She still suffers from stiffness and intermittent knee pain after finishing her TB therapy.

\section{Conclusion}

Our case highlights the importance of recognizing TB arthritis early when the clinical and epidemiological risks exist. Proceeding with appropriate diagnostic tests early on would help in avoiding any delay in diagnosis. The diagnosis of TB arthritis can be masked by the presence of a superimposed bacterial pathogen.

\section{Conflict of Interest}

The author has no conflict of interest. 


\section{Disclosure}

The author did not receive any commercial or financial support for this study. The author has no financial interest in any of the products, devices, or drugs mentioned in this article.

\section{Ethical Approval}

\section{Obtained.}

\section{References}

[1] Peto HM, Pratt RH, Harrington TA, LoBue PA, Armstrong LR. Epidemiology of extrapulmonary tuberculosis in the United States, 1993-2006. Clin Infect Dis 2009; 49(9): 13501357.

[2] Chen ST, Zhao LP, Dong WJ, Gu YT, Li YX, Dong LL, Ma YF, Qin SB, Huang HR. The clinical features and bacteriological characterizations of bone and joint tuberculosis in China. Sci Rep 2015; 5: 11084.

[3] Hodgson SP, Ormerod LP. Ten-year experience of bone and joint tuberculosis in Blackburn 1978-1987. J R Coll Surg Edinb 1990; 35(4): 259-262.

[4] Moriyama $Y$, Sono $Y$, Nishioka H. Tuberculous arthritis of the hip with Staphylococcus aureus superinfection. J Infect Chemother 2016; 22(11): 752-754.

[5] Opara TN, Gupte CM, Liyanage SH, Poole S, Beverly MC. Tuberculous arthritis of the knee with Staphylococcus superinfection. J Bone Joint Surg Br 2007; 89(5): 664-666.

[6] Walter ND, Painter J, Parker M, Lowenthal P, Flood J, Fu Y, Asis R, Reves R; Tuberculosis Epidemiologic Studies Consortium. Persistent latent tuberculosis reactivation risk in United States immigrants. Am J Respir Crit Care Med 2014; 189(1): 88-95.

[7] Enarson DA, Fujii M, Nakielna EM, Grzybowski S. Bone and joint tuberculosis: a continuing problem. Can Med Assoc J 1979; 120(2): 139-145.

[8] Walker GF. Failure of early recognition of skeletal tuberculosis. Br Med J 1968; 1(5593): 682-683.

[9] Narasimhan P, Wood J, Macintyre CR, Mathai D. Risk factors for tuberculosis. Pulm Med 2013; 2013: 828939.

[10] [No authors listed]. "Global Tuberculosis Report." World Health Organization. Accessed December 10, 2017. http:// www.who.int/tb/publications/global_report/en/.

[11] Lewinsohn DM, Leonard MK, LoBue PA, Cohn DL, Daley CL, Desmond E, Keane J, Lewinsohn DA, Loeffler AM, Mazurek GH, O'Brien RJ, Pai M, Richeldi L, Salfinger M, Shinnick TM, Sterling TR, Warshauer DM, Woods GL. Official American Thoracic Society/Infectious Diseases Society of America/ Centers for Disease Control and Prevention Clinical Practice Guidelines: Diagnosis of Tuberculosis in Adults and Children. Clin Infect Dis 2017; 64(2): 111-115.
[12] Hoffman EB, Allin J, Campbell JA, Leisegang FM. Tuberculosis of the knee. Clin Orthop Relat Res 2002; (398): 100-106. 


\section{البكتيريا المكورة العنقودية الذهبية تثنترك مع السل في مرض التهاب المفاصل}

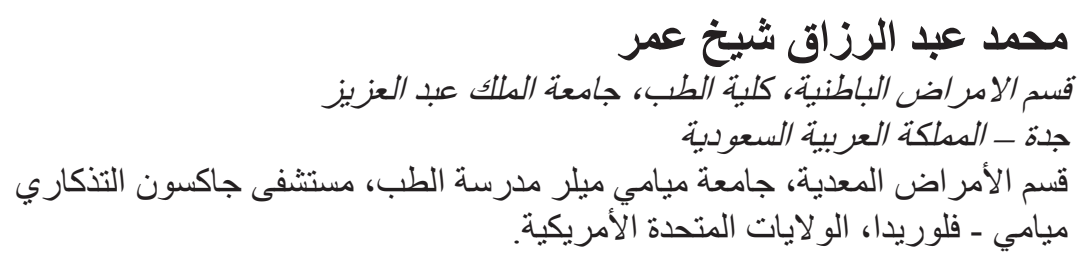

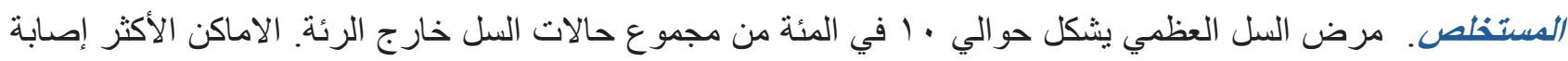

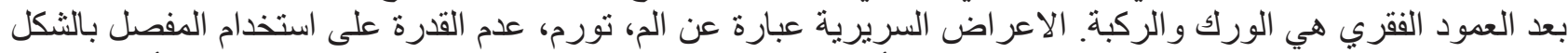

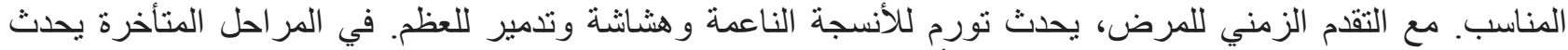

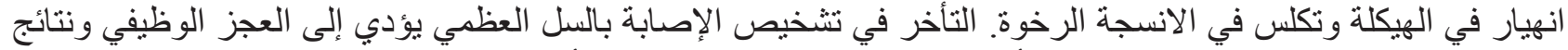

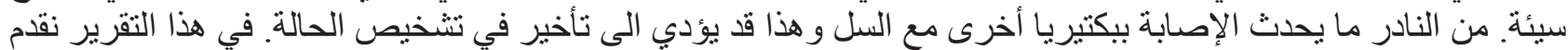

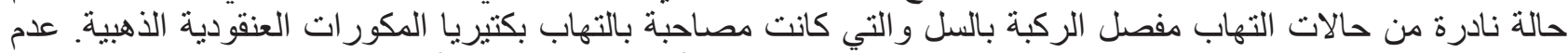

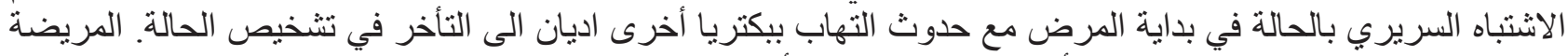

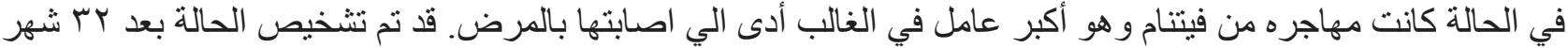
من بداية اعر اض المرض. الاشتنباه السريري بالحالة في بداية الاعر اض يساعد في تشخيص المرض في مرحلة مبكرة عن طريق طلب التحاليل اللازمة. 